International Journal of Applied Mathematical Research, 1 (3) (2012) 314-322

(C)Science Publishing Corporation

www.sciencepubco.com/index.php/IJAMR

\title{
Oscillation Criteria Of Second Order Nonlinear Neutral Differential Equations
}

\author{
A.A.Soliman, R.A.Sallam, A.M.Hassan \\ Department of Mathematics, Faculty of Science,Benha University, \\ Benha-Kalubia 13518, Egypt \\ Email:a_a_soliman@hotmail.com \\ Department of Mathematics, Faculty of Science,Monoufia University, \\ Shibin EL-Koom, Egypt \\ Email:ragaasallam@yahoo.com \\ Department of Mathematics, Faculty of Science,Benha University, \\ Benha-Kalubia 13518, Egypt \\ Email:ahmed.mohamed@fsc.bu.edu.eg
}

\begin{abstract}
This paper is concerned with the oscillation of second-order nonlinear neutral differential equations of the form

$$
\left[r(t)\left[(x(t)+p(t) x(\sigma(t)))^{\prime}\right]^{\gamma}\right]^{\prime}+f(t, x(\tau(t)))=0,
$$

by using a generalized Riccati's technique and integral averaging technique, we establish new oscillation results which handle some cases not covered by known criteria.
\end{abstract}

Keywords: Oscillation, second order, Nonlinear equation, Neutral type.

\section{Introduction}

In this paper, we consider the oscillatory behavior of solutions of second-order nonlinear neutral differential equations of the form

$$
\left[r(t)\left[(x(t)+p(t) x(\sigma(t)))^{\prime}\right]^{\gamma}\right]^{\prime}+f(t, x(\tau(t)))=0, \quad t \geq t_{0}
$$

In this paper, we assume that 
$\left(I_{1}\right) r(t) \in C^{1}\left(\left[t_{0}, \infty\right),(0, \infty)\right), r^{\prime}(t)>0, \int_{t_{0}}^{\infty} r(s)^{\frac{-1}{\gamma}} d s=\infty ;$

$\left(I_{2}\right) \quad 0 \leq p(t) \leq 1$

$\left(I_{3}\right) \sigma(t) \in C\left(\left[t_{0}, \infty\right), R\right), \tau(t) \in C^{1}\left(\left[t_{0}, \infty\right), R\right) ; \sigma(t) \leq t ; \tau(t) \leq t ;$ $\lim _{t \rightarrow \infty} \sigma(t)=\infty ; \lim _{t \rightarrow \infty} \tau(t)=\infty ; \sigma \circ \tau=\tau \circ \sigma ;$

$\left(I_{4}\right) \gamma$ is a quotient of odd positive integers;

( $\left.I_{5}\right) f(t, x(\tau(t))) \in C\left(\left[t_{0}, \infty\right) \times R, R\right)$ and there exists $q(t) \in C\left(\left[t_{0}, \infty\right), R^{+}\right)$ such that $|x f(t, x)| \geq q(t)|x|^{\gamma}$.

We set $z(t)=x(t)+p(t) x(\sigma(t))$, by a solution of Eq.(1) we mean a function $x(t) \in C\left(\left[T_{x}, \infty\right)\right), \quad T_{x} \geq t_{0}$, which has the property $r(t) z^{\prime}(t) \in C^{1}([T x, \infty))$ and satisfies (1) on $\left[T_{x}, \infty\right)$. we consider only those solutions $x(t)$ of $(1)$ which satisfy $\sup \{|x(t)|: t \geq T\}>0$ for all $T \geq T_{x}$. we assume that (1) possesses such a solution. a solution of (1) is called oscillatory if it has arbitrarily large zeros on $\left[T_{x}, \infty\right)$ and otherwise, it is said to be nonoscillatory. Eq.(1) Itself is said to be oscillatory if all of its solutions are oscillatory.

The second order equations have the applications in various problems of physics, biology, and economy. Therefore, there is constant interest in obtaining sufficient conditions for the oscillation or nonoscillation of the solutions of various types of the second order equations. See e.g. papers [1-11].

The oscillation problem for nonlinear such as

$$
\left[r(t)[x(t)+p(t) x(\tau(t))]^{\prime}\right]^{\prime}+q(t) x(\sigma(t))=0,
$$

has been studied in [2] by using new comparison theorems, that enabled them to reduce the problem from second equation order to first order equation. as well as for nonlinear equation

$$
\left[r(t)[x(t)+p(t) x(\tau(t))]^{\prime}\right]^{\prime}+q(t) f(x(\sigma(t)))=0,
$$

has been studied in [11]

In this paper we will use a generalized Riccati's technique and Integral averaging techniques, this method used in [10].

\section{Main Results}

First, we give two lemmas which will be used in the following results.

Lemma 2.1. Let conditions $\left(I_{1}\right)-\left(I_{5}\right)$ hold. If $x(t)$ is an eventually positive solution of (1), then there exists $t_{1} \in\left[t_{0}, \infty\right)$ sufficiently large such that $z(t)>$ $0 ; z^{\prime}(t)>0 ; z^{\prime \prime}(t)<0$ for all $t \in\left[t_{1}, \infty\right)$. 
Proof. Since $x(t)$ is an eventually positive solution of (1), then by $\left(I_{3}\right)$ there exists $t_{1} \in\left[t_{0}, \infty\right)$ such that

$$
x(t)>0, x(\sigma(t))>0 \text { and } x(\tau(t))>0 \quad \text { for } t \geq t_{1},
$$

from (1) and the assumption we see that $z(t) \geq x(t)>0$ and we have

$$
\left(r(t)\left[z^{\prime}(t)\right]^{\gamma}\right)^{\prime} \leq-q(t) x^{\gamma}(\tau(t))<0 \quad \text { forall } \quad t \geq t_{1},
$$

which implies that $\left(r(t)\left[z^{\prime}(t)\right]^{\gamma}\right)$ is decreasing on $[t, \infty)$. we claim that $r(t)\left[z^{\prime}(t)\right]^{\gamma}>0$ on $\left[t_{1}, \infty\right)$. Assume not, there is a $t_{2} \in\left[t_{1}, \infty\right)$. Such that $r\left(t_{2}\right)\left[z^{\prime}\left(t_{2}\right)\right]^{\gamma}<0$.

Since

$$
\begin{aligned}
\left(r(t)\left[z^{\prime}(t)\right]^{\gamma}\right) & \leq\left(r\left(t_{2}\right)\left[z^{\prime}\left(t_{2}\right)\right]^{\gamma}\right) \quad \text { for all } \quad t \geq t_{2} \\
z^{\prime}(t) & \leq\left(r\left(t_{2}\right)\right)^{\frac{1}{\gamma}} z^{\prime}\left(t_{2}\right)(r(t))^{\frac{-1}{\gamma}} .
\end{aligned}
$$

Integrating from $t_{2}$ to $t$

$$
\begin{aligned}
z(t)-z\left(t_{2}\right) & \leq \int_{t_{2}}^{t}\left(r\left(t_{2}\right)\right)^{\frac{1}{\gamma}} z^{\prime}\left(t_{2}\right)(r(s))^{\frac{-1}{\gamma}} d s \\
& \leq\left(r\left(t_{2}\right)\right)^{\frac{1}{\gamma}} z^{\prime}\left(t_{2}\right) \int_{t_{2}}^{t}(r(s))^{\frac{-1}{\gamma}} d s \\
z(t) & \leq z\left(t_{2}\right)+\left(r\left(t_{2}\right)\right)^{\frac{1}{\gamma}} z^{\prime}\left(t_{2}\right) \int_{t_{2}}^{t}(r(s))^{\frac{-1}{\gamma}} d s,
\end{aligned}
$$

from $\left(I_{1}\right)$ and as $t \rightarrow \infty ; z(t) \rightarrow-\infty$ which contradiction with the fact $z(t)>0$, this implies that $r(t)\left[z^{\prime}(t)\right]^{\gamma}>0$ and $z^{\prime}(t)>0$ on $[t, \infty)$.

To prove that $z^{\prime \prime}(t)<0$

from $(2)$ and $\left(I_{1}\right)$, we have

$$
\begin{aligned}
\left(r(t)\left[z^{\prime}(t)\right]^{\gamma}\right)^{\prime} & <0 \\
r^{\prime}(t)\left[z^{\prime}(t)\right]^{\gamma}+\gamma r(t)\left[z^{\prime}(t)\right]^{\gamma-1} z^{\prime \prime}(t) & <0,
\end{aligned}
$$

$z^{\prime \prime}(t)<0$. This completes the proof.

Lemma 2.2. Let $g(u)=B u-A u^{\frac{\gamma+1}{\gamma}}$, where $A>0$ and $B$ are constants, $\gamma$ is a quotient of odd positive integers. Then $g$ attains its maximum value on $R$ at $u^{*}=\frac{\gamma^{\gamma} B^{\gamma}}{A^{\gamma}(\gamma+1)^{\gamma}}$ and $\max (g)=\frac{\gamma^{\gamma}}{(\gamma+1)^{\gamma+1}} \frac{B^{\gamma+1}}{A^{\gamma}}$.

Proof. From hypotheses of Lemma 2.2

$$
g^{\prime}(u)=B-\frac{\gamma+1}{\gamma} A u^{\frac{1}{\gamma}},
$$


to obtain the max and min value of $g(u)$ we put $g^{\prime}(u)=0$

$$
u^{*}=\frac{\gamma^{\gamma} B^{\gamma}}{A^{\gamma}(\gamma+1)^{\gamma}}
$$

since $g^{\prime \prime}\left(u^{*}\right)<0$, then $g(u)$ attains to the max value on $R$ at $u^{*}$.i.e $g\left(u^{*}\right)$ is the maximum value of $g(u)$

$$
g\left(u^{*}\right)=\frac{\gamma^{\gamma}}{(\gamma+1)^{\gamma+1}} \frac{B^{\gamma+1}}{A^{\gamma}}
$$

and we can write the inequality

$$
B u-A u^{\frac{\gamma+1}{\gamma}}<\frac{\gamma^{\gamma}}{(\gamma+1)^{\gamma+1}} \frac{B^{\gamma+1}}{A^{\gamma}}
$$

Theorem 2.3. Assume that $\left(I_{1}\right)-\left(I_{5}\right)$ hold, and assume that there exists a positive differentiable function $\rho(t)$ such that $\rho^{\prime}(t)>0, \tau^{\prime}(t)>0, \gamma \geq 1$

$$
\limsup _{t \rightarrow \infty} \int_{t_{0}}^{t}\left[Q(s) \rho(s)-\frac{1}{(\gamma+1)^{\gamma+1}} \frac{\left(\rho^{\prime}(s)\right)^{\gamma+1} r(\tau(s))}{\rho^{\gamma}(s)\left(\tau^{\prime}(s)\right)^{\gamma}}\right] d s=\infty
$$

where $Q(t)=q(t)[1-p(\tau(t))]^{\gamma}$. Then equation (1) is oscillatory.

Proof. Suppose that $x(t)$ be a nonoscillatory solution. with out loss of generality we may assume that $x(t)$ is positive, then there exists $t_{1} \geq t_{0}$ sufficiently large such that $x(t)>0, x(\sigma(t))>0, x(\tau(t))>0$.

Since

$$
\left[r(t)\left[z^{\prime}(t)\right]^{\gamma}\right]^{\prime}+f(t, x(\tau(t)))=0
$$

from $\left(I_{5}\right)$

$$
\left[r(t)\left[z^{\prime}(t)\right]^{\gamma}\right]^{\prime} \leq-q(t) x^{\gamma}(\tau(t))
$$

from hypotheses, we obtain

$$
\begin{aligned}
x(t) & =z(t)-p(t) x(\sigma(t)) \\
x(\tau(t)) & =z(\tau(t))-p(\tau(t)) x(\sigma(\tau(t))) \\
& \geq z(\tau(t))-p(\tau(t)) x(\tau(t)) \\
& \geq[1-p(\tau(t))] z(\tau(t)),
\end{aligned}
$$

from (2) and (3), we get

$$
\left[r(t)\left[z^{\prime}(t)\right]^{\gamma}\right]^{\prime} \leq-q(t)[1-p(\tau(t))]^{\gamma} z^{\gamma}(\tau(t)) .
$$


Now define $\omega(t)$ be a positive differentiable function on the form

$$
\begin{gathered}
\omega(t)=\rho(t) \frac{r(t)\left[z^{\prime}(t)\right]^{\gamma}}{z^{\gamma}(\tau(t))} \\
\omega^{\prime}(t)=\rho^{\prime}(t) \frac{r(t)\left[z^{\prime}(t)\right]^{\gamma}}{z^{\gamma}(\tau(t))}+\rho(t) \frac{\left[r(t)\left[z^{\prime}(t)\right]^{\gamma}\right]^{\prime}}{z^{\gamma}(\tau(t))}-\gamma \rho(t) \frac{r(t)\left[z^{\prime}(t)\right]^{\gamma} z^{\prime}(\tau(t)) \tau^{\prime}(t)}{z^{\gamma+1}(\tau(t))}
\end{gathered}
$$

from (4), we have

$$
\omega^{\prime}(t) \leq \frac{\rho^{\prime}(t)}{\rho(t)} \omega(t)-\rho(t) q(t)[1-p(\tau(t))]^{\gamma} z^{\gamma}(\tau(t))-\gamma \omega(t) \frac{z^{\prime}(\tau(t)) \tau^{\prime}(t)}{z(\tau(t))},
$$

from Lemma 2.1

$$
r(t)\left[z^{\prime}(t)\right]^{\gamma} \leq r(\tau(t))\left[z^{\prime}(\tau(t))\right]^{\gamma}
$$

from (5) and (6),

$$
\begin{aligned}
\omega^{\prime}(t) & \leq \frac{\rho^{\prime}(t)}{\rho(t)} \omega(t)-\rho(t) q(t)[1-p(\tau(t))]^{\gamma}-\gamma \omega(t) \frac{z^{\prime}(t)(r(t))^{\frac{1}{\gamma}} \tau^{\prime}(t)}{z(\tau(t))(r(\tau(t)))^{\frac{1}{\gamma}}} \\
& \leq \frac{\rho^{\prime}(t)}{\rho(t)} \omega(t)-\rho(t) Q(t)-\gamma \omega^{\frac{\gamma}{\gamma+1}}(t) \frac{\tau^{\prime}(t)}{\rho^{\frac{1}{\gamma}}(t)(r(\tau(t)))^{\frac{1}{\gamma}}},
\end{aligned}
$$

put $Q(t)=q(t)[1-p(\tau(t))]^{\gamma}, B=\frac{\rho^{\prime}(t)}{\rho(t)}, A=\frac{\tau^{\prime}(t)}{\rho^{\frac{1}{\gamma}}(t)(r(\tau(t)))^{\frac{1}{\gamma}}}$

$$
\omega^{\prime}(t) \leq-\rho(t) Q(t)+B \omega(t)-A \omega^{\frac{\gamma}{\gamma+1}}(t)
$$

by using Lemma 2.2

$$
\begin{aligned}
\omega^{\prime}(t) & \leq-\rho(t) Q(t)+\frac{\gamma^{\gamma}}{(\gamma+1)^{\gamma+1}} \frac{B^{\gamma+1}}{A^{\gamma}} \\
& \leq-\rho(t) Q(t)+\frac{\gamma^{\gamma}}{(\gamma+1)^{\gamma+1}} \frac{\left(\frac{\rho^{\prime}(t)}{\rho(t)}\right)^{\gamma+1}}{\left(\frac{\gamma \tau^{\prime}(t)}{(r(\tau(t)))^{\frac{1}{\gamma}} \rho^{\frac{1}{\gamma}}}\right)^{\gamma}} \\
& \leq-\rho(t) Q(t)+\frac{1}{(\gamma+1)^{\gamma+1}} \frac{\left(\rho^{\prime}(t)\right)^{\gamma+1}}{\rho^{\gamma}(t)} \frac{r(\tau(t))}{\left(\tau^{\prime}(t)\right)^{\gamma}}
\end{aligned}
$$

Integrating (8) from $t_{1}$ to $t$

$$
\omega(t)-\omega\left(t_{1}\right) \leq-\int_{t_{1}}^{t}\left[\rho(s) Q(s)-\frac{1}{(\gamma+1)^{\gamma+1}} \frac{\left(\rho^{\prime}(s)\right)^{\gamma+1}}{\rho^{\gamma}(s)} \frac{r(\tau(s))}{\left(\tau^{\prime}(s)\right)^{\gamma}}\right] d s,
$$

taking the $\lim \sup _{t \rightarrow \infty} ; \omega(t) \rightarrow-\infty$ which contradicts with $\omega(t)$ positive, then Eq.(1)is oscillatory. 
Remark 2.4. the above theorem is more general of theorem 3.1 in [10].

Following [10],Define $D=\{(t, s): t \geq s \geq 0\}$ and $H=\left\{H(t, s) \in C^{1}\left(D, R_{+}\right): H(t, t)=0, H(t, s)>0 ; \frac{\partial H}{\partial s} \geq 0\right.$ for all $t \geq s \geq$ $0\}$

Theorem 2.5. Assume that $\left(I_{1}\right)-\left(I_{5}\right)$ hold, and assume that there exists a positive differentiable function $\rho(t)$ and a function $H(t, s) \in H$ such that

$$
\limsup _{t \rightarrow \infty} \frac{1}{H\left(t, t_{0}\right)} \int_{t_{0}}^{t}\left[H(t, s) Q(s) \rho(s)-\frac{[C(t, s)]}{(\gamma+1)^{\gamma+1}} \frac{\rho(s) r(\tau(s))}{\left(\tau^{\prime}(s)\right)^{\gamma} H^{\gamma}(t, s)}\right] d s=\infty
$$

where $C(t, s)=\frac{\partial H}{\partial s}+H(t, s) \frac{\rho^{\prime}(s)}{\rho(s)}$. Then Eq.(1)is oscillatory.

Proof. Suppose that $x(t)$ be a positive solution of Eq.(1). from (theorem 2.3) and Eq.(7) by multiplying two sides by $H(t, s)$

$$
\begin{aligned}
& H(t, s) \omega^{\prime}(t) \leq H(t, s) \frac{\rho^{\prime}(t)}{\rho(t)} \omega(t)-H(t, s) \rho(t) Q(t)-\gamma H(t, s) \omega^{\frac{\gamma}{\gamma+1}}(t) \frac{\tau^{\prime}(t)}{\rho^{\frac{1}{\gamma}}(t)(r(\tau(t)))^{\frac{1}{\gamma}}} \\
& \begin{aligned}
H(t, s) \rho(t) Q(t) \leq-H(t, s) \omega^{\prime}(t) \\
\quad+H(t, s) \frac{\rho^{\prime}(t)}{\rho(t)} \omega(t)-\gamma H(t, s) \omega^{\frac{\gamma}{\gamma+1}}(t) \frac{\tau^{\prime}(t)}{\rho^{\frac{1}{\gamma}}(t)(r(\tau(t)))^{\frac{1}{\gamma}}} .
\end{aligned}
\end{aligned}
$$

Integrating Eq.(9) from $t_{1}$ to $t$

$$
\begin{aligned}
\int_{t_{1}}^{t} H(t, s) \rho(s) Q(s) d s \leq & -\int_{t_{1}}^{t} H(t, s) \omega^{\prime}(s) d s+\int_{t_{1}}^{t} H(t, s) \frac{\rho^{\prime}(s)}{\rho(s)} \omega(s) d s \\
& -\int_{t_{1}}^{t} \gamma H(t, s) \omega^{\frac{\gamma}{\gamma+1}}(t) \frac{\tau^{\prime}(s)}{\rho^{\frac{1}{\gamma}}(s)(r(\tau(s)))^{\frac{1}{\gamma}}} d s
\end{aligned}
$$

since

$$
\int_{t_{1}}^{t} H(t, s) \omega^{\prime}(s) d s=-H\left(t, t_{1}\right) \omega\left(t_{1}\right)-\int_{t_{1}}^{t} \frac{\partial H}{\partial s} \omega(s) d s,
$$

from (10) and (11), we get

$$
\begin{aligned}
\int_{t_{1}}^{t} H(t, s) \rho(s) Q(s) d s \leq & H\left(t, t_{1}\right) \omega\left(t_{1}\right)+\int_{t_{1}}^{t} \frac{\partial H}{\partial s} \omega(s) d s+\int_{t_{1}}^{t} H(t, s) \frac{\rho^{\prime}(s)}{\rho(s)} \omega(s) d s \\
& -\int_{t_{1}}^{t} \gamma H(t, s) \omega^{\frac{\gamma}{\gamma+1}}(t) \frac{\tau^{\prime}(s)}{\rho^{\frac{1}{\gamma}}(s)(r(\tau(s)))^{\frac{1}{\gamma}}} d s,
\end{aligned}
$$




$$
\begin{aligned}
\int_{t_{1}}^{t} H(t, s) \rho(s) Q(s) d s \leq & H\left(t, t_{1}\right) \omega\left(t_{1}\right)+\int_{t_{1}}^{t}\left[\frac{\partial H}{\partial s}+H(t, s) \frac{\rho^{\prime}(s)}{\rho(s)}\right] \omega(s) d s \\
& -\int_{t_{1}}^{t} \gamma H(t, s) \omega^{\frac{\gamma}{\gamma+1}}(t) \frac{\tau^{\prime}(s)}{\rho^{\frac{1}{\gamma}}(s)(r(\tau(s)))^{\frac{1}{\gamma}}} d s,
\end{aligned}
$$

put $B=\left[\frac{\partial H}{\partial s}+H(t, s) \frac{\rho^{\prime}(s)}{\rho(s)}\right]$ and $A=\gamma H(t, s) \frac{\tau^{\prime}(s)}{\rho^{\frac{1}{\gamma}}(s)(r(\tau(s)))^{\frac{1}{\gamma}}}$

from lemma 2.2 and (12)

$$
\begin{aligned}
\int_{t_{1}}^{t} H(t, s) \rho(s) Q(s) d s & \leq H\left(t, t_{1}\right) \omega\left(t_{1}\right)+\int_{t_{1}}^{t} B \omega(s)-A \omega^{\frac{\gamma}{\gamma+1}}(s) d s \\
& \leq H\left(t, t_{1}\right) \omega\left(t_{1}\right)+\int_{t_{1}}^{t} \frac{\gamma^{\gamma}}{(\gamma+1)^{\gamma+1}} \frac{B^{\gamma+1}}{A^{\gamma}} d s \\
& \leq H\left(t, t_{1}\right) \omega\left(t_{1}\right)+\int_{t_{1}}^{t} \frac{\gamma^{\gamma}}{(\gamma+1)^{\gamma+1}} \frac{\left[\frac{\partial H}{\partial s}+H(t, s) \frac{\rho^{\prime}(s)}{\rho(s)}\right]^{\gamma+1}}{\left[\gamma(t, s) \frac{\tau^{\prime}(s)}{\rho^{\frac{1}{\gamma}}(s)(r(\tau(s)))^{\frac{1}{\gamma}}}\right]^{\gamma}} d s \\
& \leq H\left(t, t_{1}\right) \omega\left(t_{1}\right)+\int_{t_{1}}^{t} \frac{\gamma^{\gamma}}{(\gamma+1)^{\gamma+1}} \frac{[C(t, s)]^{\gamma+1} r(\tau(s)) \rho(s)}{\left[\gamma \tau^{\prime}(s) H(t, s)\right]^{\gamma}} d s,
\end{aligned}
$$

where $C(t, s)=\frac{\partial H}{\partial s}+H(t, s) \frac{\rho^{\prime}(s)}{\rho(s)}$.

$$
\frac{1}{H\left(t, t_{1}\right)} \int_{t_{1}}^{t} H(t, s) \rho(s) Q(s)-\frac{1}{(\gamma+1)^{\gamma+1}} \frac{[C(t, s)]^{\gamma+1} r(\tau(s)) \rho(s)}{\left[\tau^{\prime}(s) H(t, s)\right]^{\gamma}} d s \leq \omega\left(t_{1}\right) .
$$

Taking limsup of the both sides of the above inequality as $t \rightarrow \infty$ we obtain contradiction with the assumptions of theorem 2.5. Then Eq.(1) is oscillatory.

Remark 2.6. the above theorem is more general of theorem 3.2 of [10]

\section{Examples}

\section{Example 1. Consider}

$$
\left[\sqrt{t}\left[x(t)+\left(1-\frac{1}{t^{\alpha}}\right) x(t-\delta)\right]^{\prime}\right]^{\prime}+\frac{\vartheta}{4 t^{\frac{3}{2}-\beta}} x(\lambda t)=0 . \quad t \geq t_{0}
$$

where $\vartheta>0, \quad \alpha \geq 0, \quad 0<\beta<1, \quad 0<\lambda<1, \quad t \geq 1$.

Note that $\gamma=1, r(t)=\sqrt{t}>0, \int_{t_{0}}^{t} s^{\frac{-1}{2}} d s=\infty, p(t)=\left(1-\frac{1}{t^{\alpha}}\right), q(t)=\frac{\vartheta}{4 t^{\frac{3}{2}-\beta}}$, 
$\tau(t)=\lambda t, \sigma(t)=t-\delta$

choose $\rho(t)=r(\tau(t))=\sqrt{\lambda t}, \quad \rho^{\prime}(t)=\frac{\lambda}{2 \sqrt{\lambda t}}$.

$Q(t)=\frac{\vartheta}{4 t^{\frac{3}{2}-\beta}}\left(\frac{1}{\lambda t}\right)^{\alpha}, \rho(t) Q(t)=\frac{\vartheta}{4 t^{\frac{3}{2}-\beta}}\left(\frac{1}{\lambda t}\right)^{\alpha} \sqrt{\lambda t}$

by applying theorem 2.3

$$
\begin{aligned}
\int_{t_{0}}^{t}\left[Q(s) \rho(s)-\frac{1}{(\gamma+1)^{\gamma+1}} \frac{\left(\rho^{\prime}(s)\right)^{\gamma+1} r(\tau(s))}{\rho^{\gamma}(s)\left(\tau^{\prime}(s)\right)^{\gamma}}\right] d s & =\int_{t_{0}}^{t}\left[\frac{\vartheta}{4 t^{\frac{3}{2}-\beta}}\left(\frac{1}{\lambda t}\right)^{\alpha} \sqrt{\lambda t}-\frac{\lambda^{2} \sqrt{\lambda s}}{4 \lambda^{2} s}\right] d s \\
& =\frac{\vartheta \lambda^{\frac{1-2 \alpha}{2}}}{4(\beta-\alpha)} t^{(\beta-\alpha)}-\left.\frac{\sqrt{\lambda s}}{2}\right|_{t_{0}} ^{t}
\end{aligned}
$$

from (14)

$$
\limsup _{t \rightarrow \infty} \int_{t_{0}}^{t}\left[Q(s) \rho(s)-\frac{1}{(\gamma+1)^{\gamma+1}} \frac{\left(\rho^{\prime}(s)\right)^{\gamma+1} r(\tau(s))}{\rho^{\gamma}(s)\left(\tau^{\prime}(s)\right)^{\gamma}}\right] d s=\infty
$$

Then the equation (13) is oscillatory.

Note that Example 1. essentially and extends Example 4.1. of [5]

Example 2. consider the following differential equation

$$
\left[\left[\left(x(t)+\frac{1}{2} x(\sigma(t))\right)^{\prime}\right]^{3}\right]^{\prime}+\frac{a}{t^{2}} x^{3}(\sqrt{t})=0 \quad, t \geq t_{0}
$$

where $\sigma(t) \in C\left(\left[t_{0}, \infty\right), R\right), \sigma(t) \leq t$.

Note that $p(t)=\frac{1}{2}, q(t)=\frac{a}{t^{2}}, a>0, r(t)=1, \gamma=3, \tau(t)=\sqrt{t}$.

choose $\rho(t)=t, \rho^{\prime}(t)=1$

since $Q(t)=q(t)[1-p(\tau(t))]^{\gamma}=\frac{a}{8 t^{3}}$

by applying theorem 2.3

$$
\begin{aligned}
\int_{t_{0}}^{t}\left[Q(s) \rho(s)-\frac{1}{(\gamma+1)^{\gamma+1}} \frac{\left(\rho^{\prime}(s)\right)^{\gamma+1} r(\tau(s))}{\rho^{\gamma}(s)\left(\tau^{\prime}(s)\right)^{\gamma}}\right] d s & =\int_{t_{0}}^{t}\left[\frac{a}{8 s}-\frac{1}{4^{4} s^{3}\left(\frac{1}{2 \sqrt{s}}\right)^{3}}\right] d s \\
= & \frac{1}{16}\left(\frac{1}{\sqrt{t}}-\frac{1}{\sqrt{t_{0}}}+2 a \log [t]-2 a \log \left[t_{0}\right]\right)
\end{aligned}
$$

from (16)

$$
\limsup _{t \rightarrow \infty} \int_{t_{0}}^{t}\left[Q(s) \rho(s)-\frac{1}{(\gamma+1)^{\gamma+1}} \frac{\left(\rho^{\prime}(s)\right)^{\gamma+1} r(\tau(s))}{\rho^{\gamma}(s)\left(\tau^{\prime}(s)\right)^{\gamma}}\right] d s=\infty
$$

Then the equation (15) is oscillatory. 


\section{References}

[1] R. P. AGARWAL,SHIOW-LING SHIEH AND CHEH-CHIH YEH, Oscillation Criteria for Second-Order Retarded Differential Equations, Math. Comput. Modelling Vol. 26, No. 4, pp. 1-11.

[2] B.Baculkova, J.Dzurina, Oscillation theorems for second order neutral differential equations, Computers and Mathematics with Applications 61 (2011) 94-99.

[3] J. Dzurina, I.P. Stavroulakis, Oscillation criteria for second-order delay differential equations, Applied Mathematics and Computation 140 (2003) 445-453.

[4] J.K. Hale, Theory of Functional Differential Equations, Springer-Verlag, New York, 1977.

[5] Jiu-Gang Dong, Oscillation behavior of second order nonlinear neutral differential equations with deviating arguments, Computers and Mathematics with Applications 59 (2010) 3710-3717.

[6] Luhong Ye, Zhiting Xu, Oscillation criteria for second order quasilinear neutral delay differential equations, Applied Mathematics and Computation 207 (2009) 388-396.

[7] Mustafa Hasanbulli, Yuri V. Rogovchenko, Oscillation criteria for second order nonlinear neutral differential equations, Applied Mathematics and Computation 215 (2010) 4392-4399.

[8] Run Xu, Fanwei Meng, Some new oscillation criteria for second order quasi-linear neutral delay differential equations, Appl. Math. Comput. 182 (2006) 797-803.

[9] Run Xu, Fanwei Meng, Oscillation criteria for second order quasi-linear neutral delay differential equations, Appl. Math. Comput. 192 (2007) 216222.

[10] Shao-Yan Zhang, Qi-Ru Wang, Oscillation of second-order nonlinear neutral dynamic equations on time scales, Applied Mathematics and Computation 216 (2010) 2837-2848.

[11] Yuan Gong Sun, Fan Wei Meng, Note on the paper of Dzurina and Stavroulakis, Appl. Math. Comput. 174 (2006) 1634-1641. 